\title{
LA REVISTA INDEX DE ENFERMERÍA: INFORMACIÓN BIBLIOGRÁFICA, INVESTIGACIÓN Y HUMANIDADES
}

\author{
MANuel AmezcuA \\ Director Revista Index de Enfermeria
}

\section{RESUMEN}

En este trabajo el autor describe la evolcuión de la revista "Index de Enfermería"

Palabras clave: Revistas de enfermeria, historia de la enfermeria, antropología de la enfermeria.

Palabras clave: Historia de la Enfermeria.

\section{THE INDEX OF NURSING JOURNAL: BIBLIOGRAPHIC, REARCH AND HUMANITIES}

\section{SUMMARY}

In this work the author describes the evolution of the journal "Index of nursing".

Keywords: Nursing journals, nursing history, nursing anthropology.

Keywords: Nursing history.

Es curioso, pero ha sido con motivo de esta ponencia la primera vez que el equipo de Index nos hemos puesto a pensar seriamente sobre lo que hemos hecho y por qué. Eso no quiere decir que en sus orígenes no hubiera una intencionalidad clara al plantear la revista y sus aledaños, o sea, no podemos defenderla como algo absolutamente espontáneo y circunstancial, pero sí debemos reconocer que la historia de Index es un fiel reflejo de la evolución intelectual de quienes andamos detrás de ella.

Cuando se cumplen doce años de su nacimiento, la revista Index de Enfermería sigue siendo sustancialmente lo que fue en su origen, un instrumento de apoyo dirigido principalmente a profesionales e investigadores de la enfermería, pero la afluencia de otros profesionales de disciplinas afines y colaterales, tanto en su confección como en su consumo, ha enriquecido de manera determi- nante sus contenidos hasta configurar un producto que no tenemos por menos que reconocer que es atípico. Esto hace que recibamos mensajes muy contradictorios por parte de nuestros lectores: a unos les parece un tanto singular por sus contenidos, incluso divertida por el uso que se hace del grabado como forma hegemónica de ilustración, a otros les parece en cambio aburrida por la falta de colorido, incluso tediosa al considerar que tiene demasiado texto y muy apretado, para finalmente quedarnos con los de siempre, con aquellos que terminan desde la distancia estableciendo una relación un tanto sentimental, con los claroscuros propios de las relaciones pasionales, hasta contemplar Index como una revista de culto.

Al tratarse de un proyecto más intelectual que comercial, tenemos que reconocer una cierta torpeza en asuntos tan importantes como la distribución, la circulación, la financiación, etc., lo que le provee de la calidad de empresa ruinosa y a nosotros nos libera de tener que ocuparnos de materias en las que nos sentimos inválidos. En esto sí que se ha comportado espontáneamente: Index ha crecido motu propio desde sus inicios lo justo para que pueda continuar realizándose, si bien con las escaseces de recursos que, entre otras cosas, nos obligan a tener permanentemente despierto el ingenio. Pero a pesar de todo nunca perdemos la esperanza de que un acercamiento intelectual hacia la mayoría de nuestros colegas, o de ellos hacia nosotros, nos libere de la condición de marginalidad (residualidad) en que generalmente nos sentimos inmersos.

En esta ponencia realizaré un recorrido un tanto personal por la historia de nuestra revista 
para terminar describiendo lo que es Index de Enfermería en la actualidad, sus valores y principios, así como nuestras expectativas para el futuro. Pero antes es necesario aclarar que para nosotros Index es algo más que una revista. Se trata de un producto que se enmarca en un proyecto mucho más ambicioso donde confluyen varias cuestiones:

- la necesidad de profundizar en el pensamiento enfermero con el objeto de construir referentes teóricos y metodológicos que posibiliten la aplicación de una ciencia enfermera que sea realmente útil a la sociedad,

—el trabajo de laboratorio, donde se someten a experimentación herramientas tanto para desarrollar la investigación en enfermería como para ejercer la práctica de los cuidados con unos mínimos de rigor,

- y, finalmente, la realización de acciones concretas de compromiso que permitan aplicar una buena parte de lo que somos capaces de asimilar teóricamente, con especial referencia a contextos y realidades poco favorecidas socialmente.

\section{8: LAS MOTIVACIONES}

En el año 1988 se publica el primer número de Index Comentado de Enfermería como una gaceta de información bibliográfica con un monográfico sobre la Visita Domiciliaria. Se trata de una revista producida en xerocopia y con la tirada suficiente para que alcanzase a un grupo de profesionales de la enfermería que habían recibido el encargo de poner en marcha la reforma sanitaria de Atención Primaria de Salud en una zona rural de Andalucía.

Su carácter de iniciativa institucional hizo que fuera demandada por numerosos profesionales que desde contextos parecidos veían en los repertorios bibliográficos de Index, sostenidos en apenas media docena de revistas, una oportunidad para actualizar sus conocimientos ante los nuevos retos y actividades que la reforma sanitaria les ponía delante.

Para quienes participamos en aquellos primeros momentos suponía un gesto de inconformismo ante una situación de dependencia disciplinar que se convirtió en un impedimento para progresar profesionalmente. El dedicar el primer número a profundizar en la modalidad de atención que había de identificar la enfermería comunitaria era muestra de ello.

\section{8-1990: LAS FUENTES}

Es una época de cambios en el Sistema Sanitario español, que afecta de manera sustancial a la enfermeria institucional, que va a experimentar cambios muy importantes en la delimitación de nuevos espacios de cuidados. Esta circunstancia sirve de revulsivo para las empresas editoriales especializadas que inician una desenfrenada carrera para aportar textos que sirvan de apoyo a las innovaciones en salud. En el caso de la enfermería y ante la escasez de textos se produce un cierto caos editorial: aumenta el número de revistas especializadas, se traducen textos foráneos, se realizan obras por encargo, e incluso hay un espacio para la picaresca (algunas editoriales con pocos escrúpulos aprovechan para dar salida a antiguallas de la Medicina encuadernándolas como textos de enfermería), predominando en general la mercadería sobre la racionalidad científica.

Index se consolida como un proyecto institucional que tiene como objetivo descubrir las fuentes de la enfermería para ponerlas al servicio de sí misma, y para ello adopta una serie de criterios respecto a las fuentes de información:

- Separar lo propio de lo ajeno, descartando aquellas contribuciones de realidades no extrapolables a la nuestra.

-Dignificar las fuentes propias, otorgándoles la consideración de soporte científico. 
- Considerar la importancia de la literatura gris (materiales no publicados pero de amplia distribución), ya que a menudo contienen experiencias válidas y recientes cargadas de originalidad.

Como soporte de los repertorios bibliográficos publicados en la revista se crea en formato Dbase la base de datos CUIDEN, que en la medida en que es conocida por profesionales cercanos al núcleo editor de Index se convierte progresivamente en una herramienta de búsqueda bibliográfica. Aunque predominan como principales consumidores los profesionales interesados en el cambio, aparecen otros nuevos: los investigadores.

\section{1-1997: LA INVESTIGACIÓN}

Al final de la década, cuando la sede de Index se encontraba en el Centro de Distrito Sanitario de Alcalá la Real (Jaén), se inicia un proceso de privatización y profesionalización de Index. Los titulares institucionales de los derechos de edición los ceden en favor de una Asociación de profesionales creada para gestionar el proyecto. Asi nace el Centro de Documentación en Enfermería Comunitaria (CEDEC), que en el año 1991 establece su sede en el Hospital Universitario de San Rafael de Granada y dos años más tarde, por acuerdo con la Delegación Provincial de Salud, en el Hospital de San Juan de Dios de la misma ciudad. En el año 1994 el CEDEC se convierte en "Fundación Index para el desarrollo de la Investigación en Cuidados de Salud", instalándose en su sede actual, y en 1997 obtiene el reconocimiento de Utilidad Pública por la Consejería de Educación y Ciencia de la Junta de Andalucía, que de esta manera ejerce su mecenazgo sobre la Fundación.

En el año 1992 se edita el primer número de Index de Enfermería como revista mixta (primaria y secundaria), de aparición trimestral, con tres lineas editoriales:

a) Documentación científica: editando repertorios bibliográficos de la producción enfermera en español (sección Index), repertorios bibliográficos comentados y temáticos (excerpta) y una sección dedicada al comentario crítico de libros (abstracta).

b) Apoyo a la investigación y metodologías de trabajo, a través de secciones como pliegos de estilo (redacción científica), instrumentos (herramientas de trabajo), cartilla de la salud (experiencias de educación sanitaria), etc.

c) Línea de pensamiento doctrinal centrada en la aplicación de modelos y tendencias en enfermería, y en historia y cultura de los cuidados.

Es de destacar que se mantiene en la nueva revista la idea de trabajar con temas monográficos, lo que permite fomentar el ejercicio de revisión sobre temas de candente actualidad o de un significado especial para el conocimiento de los cuidados. Hasta ahora se han publicado los siguientes monográficos:

1. El diabético: una década de cuidados en España

2. La Visita Domiciliaria: cien referencias comentadas

3. Documentación Cientifica y Salud

4-5. La Historia de los cuidados en España

6. El Sistema Informal de Cuidados

7. El arte de los autocuidados, para una teoría aplicable a todas las realidades

8-9. El anciano en casa: los cuidados y los cuidadores

10. Terapias alternativas y técnicas naturales para el cuidado

11. Registros clínicos y calidad: el reto de la enfermería convincente

12-13. El Diagnóstico de Enfermería: ¿una realidad apreciable en España?

14. Juan de Dios, un enfermero para la humanidad 15. Enfermeras en la Guerra Civil Española

16-17. Bibliometría: producción cientifica de la enfermería española.

18. Autocuidados y calidad de vida.

19. Iconografía y enfermería: un instrumento para la investigación histórica. 
20-21. Index 1988-1998: diez años de documentación enfermera en España.

22. Lactancia materna: hacia una feliz lactancia natural.

23. Mayores y sociedad: cuidados familiares y redes de apoyo

24-25. Enfermería Comunitaria: veinte años después de Alma Ata

26. Investigación participativa en Salud

27. Evidencia científica y enfermería.

28-29. Investigación cualitativa en salud.

30. La violencia contra la mujer.

31. Mutilación Genital Femenina (en prensa)

32-33. Anorexia: enfermedad y mal social (próximo)

34. Los jóvenes y la salud (próximo)

\section{DESDE EL 98: LA APLICACIÓN PRÁCTICA}

Las nuevas condiciones creadas en torno al proyecto Index (nueva fórmula jurídica, mejor infraestructura, mayor capacidad de acción) tienen como consecuencia la aplicación a la práctica de muchas de las ideas y proyectos que se habían ido acariciando en los últimos años.

La principal de ellas fue la universalización de la base de datos CUIDEN, que a través de un convenio con la empresa catalana Doc6, se logra situar en Internet, de manera que es accesible de manera gratuita por todos los usuarios de la red, o sea, puede consultarse desde cualquier parte del mundo.

Otros aspectos destacables de la nueva andadura de la Fundación, que tienen un reflejo determinante en la revista Index son los siguientes:

-La Fundación financia un proyecto de investigación para obtener indicadores bibliométricos en la producción enfermera, que nos permitió ver el comportamiento de las revistas respecto a su impacto, autoría, etc. La primera publicación de estos indicadores se realizó en el número correspondiente a la primavera-verano del 98.
-Bajo el lema de "Investigar para la acción" se pone en marcha un programa de formación de investigadores encaminado a dinamizar grupos de investigación con capacidad de gestionar proyectos aplicados a los cuidados. Para facilitar el intercambio entre investigadores se ha creó el fondo de datos "Red de Investigadores", que recoge información de grupos de investigación y proyectos realizados y en curso, que puede consultarse a través de la base de datos CUIDEN, publicándose algunos repertorios en Index, en la sección del mismo nombre.

-De manera más concreta se apuesta por la promoción de la Metodología cualitativa, a la que se le atribuyen utilidades específicas en el campo de la enfermería, en tanto permite conocer la perspectiva del paciente y su familia en el proceso salud-enfermedad. La creación del Laboratorio de Investigación Cualitativa, la realización de dos ediciones de la Reunión de Investigación Cualitativa en Salud, y la publicación de dos monográficos de la revista (Investigación participativa en Salud, Investigación cualitativa en salud), a la que se han añadido secciones específicas (Diario de campo, Retablo de las Maravillas, Historia y vida, La Mirada, etc) son una muestra de este intento. La metodología cualitativa ha posibilitado el encuentro con otra de las líneas editoriales de la revista, las humanidades en salud.

-Finalmente, la apertura dentro de la Fundación de una línea de acciones de Cooperación al desarrollo, materializada en la realización de diversos proyectos en países del tercer mundo y en la creación del Fondo Index Solidaridad, está permitiendo llevar a la práctica metodologías de trabajo basadas en la participación comunitaria y en la investigación-acción en comunidades socialmente desfavorecidas.

\section{¿CÓMO ES INDEX DE ENFERMERÍA HOY?}

Es una revista cientifica enfermera de cobertura internacional, dimensión ésta que le otorga su 
creciente circulación (en 1998 aparece como la cuarta revista de enfermería en español más citada), especialmente en el ámbito iberoamericano, y la presencia constante de autores de nacionalidades diversas.

En la última transformación de su formato la hemos subtitulado "De Información Bibliográfica, Investigación y Humanidades", pues bajo este lema se pueden enmarcar sus actuales líneas editoriales:

-Documentación (encaminada a la evidencia científica)

-Herramientas para el cuidado

-Promoción de la Investigación

-Marcos de referencia teóricos y filosóficos

-Historia e identidad de la enfermería

-Investigación cualitativa

-Humanización en salud

-Cultura de la salud

Sus contenidos según las secciones abiertas en la actualidad son los siguientes:

a) Información bibliográfica:

-Repertorios bibliográficos periódicos de la producción enfermera en español, agrupados por autores y con un índice de materias

-Abstracta: comentario crítico de libros

-Excerpta: síntesis bibliográficas comentadas sobre un tema monográfico

-Archivo: textos de interés para el conocimiento de la historia de los cuidados

-Cartilla de la salud: descripción de experiencias en educación sanitaria

-Instrumentos: descripción de herramientas para el cuidado

-La mirada: utilidad de la imagen para el conocimiento de los cuidados

-Foro científico: edición de materiales presentados en reuniones cientificas

\section{b) Investigación}

-Artículos de contenido científico: originales, revisiones, editoriales, cartas al director
-Artículos de apoyo a la investigación: Metodología de investigación, Investigación Cualitativa, Evidencia Cientifica, Pliegos de estilo (propuestas en redacción científica), Red de Investigadores

c) Humanidades

-La búsqueda (reportajes y entrevistas de personas destacadas en investigación y la práctica de los cuidados)

-Historia y vida (relatos biográficos que recogen la experiencia vivida de personas respecto de su salud)

-Diario de campo (relatos de profesionales y cuidadores que recogen su vivencia en el cuidado)

-Retablo de las maravillas (reportajes de lugares y experiencias de interés para los cuidados)

También en su confección interna, Index ha de considerarse una revista un tanto peculiar. Como muestra baste decir que casi el $40 \%$ de su contenido se elabora por la propia redacción de la revista. Al trabajar por monográficos, supone que otra parte importante del contenido se realiza por encargo de la redacción. El resto contiene las colaboraciones espontáneas de los autores.

La calidad de la revista es garantizada a través de dos mecanismos:

a) la uniformidad se consigue exigiendo a los autores que se atengan a las normas de estilo que se publican periódicamente en la revista.

b) la adecuación metodológica se busca a través del sistema de revisión por pares: cada artículo es enviado a dos árbitros que en condiciones ciegas determina el grado de adecuación del manuscrito a través de un informe estructurado que remite al director de la revista. A éste compete la decisión final de aceptación, denegación o aceptación condicionada, que le es comunicada a los autores.

El equipo de redacción se compone en la actualidad de nueve redactores, una redactora jefe, una secretaria de redacción, una documentalista y un director. Además existe otro equipo de diez 
redactores en la sede de Zaragoza y cinco corresponsales. De las líneas editoriales de la revista se cuida un consejo asesor compuesto de treinta miembros. Todos estos cargos se ejercen de manera altruista.

\section{Algunos aspectos que hemos tenido que vencer}

-La tentación de convertir el proyecto Index en una forma sectaria. La prueba de que no ha sido así es la capacidad de renovación que le ha caracterizado.

- La desidia de las instituciones que en teoría deberían apoyarlo: ni las que dicen fomentar la investigación ni las que pregonan su defensa de la enfermería se han mostrado sensibles a nuestras demandas de apoyo (especialmente financiero). Hemos recibido más apoyos morales que ayudas efectivas, y aquellos lo han mostrado especialmente otras sociedacles cientificas, algunas Escuelas de Enfermeria y algunos hospitales, donde han sido mejor acogidas las actividades de Index.

-El ansia fagocitadora de los que manejan los grandes recursos en enfermería y fuera de ella. Cada cierto tiempo se recibe una oferta de fusión o absorción de la revista, más por aprovecharse de su teórica influencia que por sensibilidad a los principios que la sustentan.

-La desidia de la mayoría de los "sabios", o sea, de aquellos que se muestran públicamente como los sostenedores de los valores ideológicos de la enfermeria, y que claramente no conocen aún la revista, o si la conocen no la leen, o si la leen no lo reconocen.

-El etnocentrismo de la enfermería: hemos tenido que romper la visión reduccionista que una buena parte de las enfermeras tienen sobre su propia disciplina para abrimos y estrechar lazos con otros saberes que también entienden de cuidados y cuidadores.

-Saber que para hacer progresar nuestro proyecto, tenemos que esperar en parte a que progrese la propia enfermería.
-Nuestra propia incapacidad para hacer de Index un proyecto económicamente rentable (aunque sí lo ha sido científicamente), lo que nos obliga a atravesar periodos de escaseces que limitan su capacidad productiva.

\section{Cosas que nos han motivado}

- Observar que el equipo se renueva y se mantiene joven, imprimiendo al proyecto el suficiente dinamismo para que las innovaciones sean siempre acogidas de buen grado.

-Que gustamos de manera especial a los jóvenes y que interesamos a los periféricos de la salud (auxiliares, sociólogos, antropólogos, historiadores, etc).

-El comprobar la fidelidad de nuestros lectores, que en una porción importante participan activamente en sus contenidos. También el comprobar que detrás de cada lector de Index se encuentra un. crítico apasionado.

-El éxito de convocatoria que siempre ha acompañado a las actividades que sostienen financieramente la revista: cursos, reuniones científicas, etc.

-La llamada de Latinoamérica, que nos muestra cada día caras diferentes de la enfermería de las que es posible aprender.

-La oportunidad de actuar como observadores de los cambios en la enfermería y la investigación. En este sentido y a través de sus compilaciones bibliográficas, Index se comporta como un registro notarial.

Valores que sustentan a Index

-La libertad: de pensamiento y de actuación.

-La innovación como constante.

-La dependencia de recursos propios

-El proyecto colectivo es más importante que las personas individuales que de él forman parte

-Los conocimientos y la experiencia generados han de utilizarse para transformar la realidad Principios 
-El español es un idioma científicamente válido (también en español se escribe la ciencia).

-La ciencia no tiene que ser necesariamente aburrida.

-Pensar en términos de cuidar puede reducirse a los límites de la ciencia o de una profesión, pero la práctica del cuidado es algo que compete a todos los ciudadanos, de los que ha de esperarse una participación responsable.

-El profesional de enfermería sólo legitima su participación en el cuidado generando conocimientos a través de la investigación y una práctica basada en la evidencia científica.

-La colaboración contribuye a generar innovaciones mucho más que las ideas.
-La cooperación con sectores desfavorecidos favorece la igualdad de los pueblos, la recuperación y revitalización de los saberes tradicionales y la cultura los dignifica.

Finalmente y en nombre de todos cuantos andamos detrás de este proyecto (o más bien realidad) sólo me queda manifestar que en Index cabe todo el mundo, quienes lo hacen y quienes lo utilizan, que muchas veces son los mismos.

Lanzamos nuestra invitación a todos los enfermeros y enfermeras de buena voluntad que crean en las posibilidades de la enfermería a que hagan útiles sus aspiraciones vinculándose a este proyecto, que es ya un proyecto de todos.

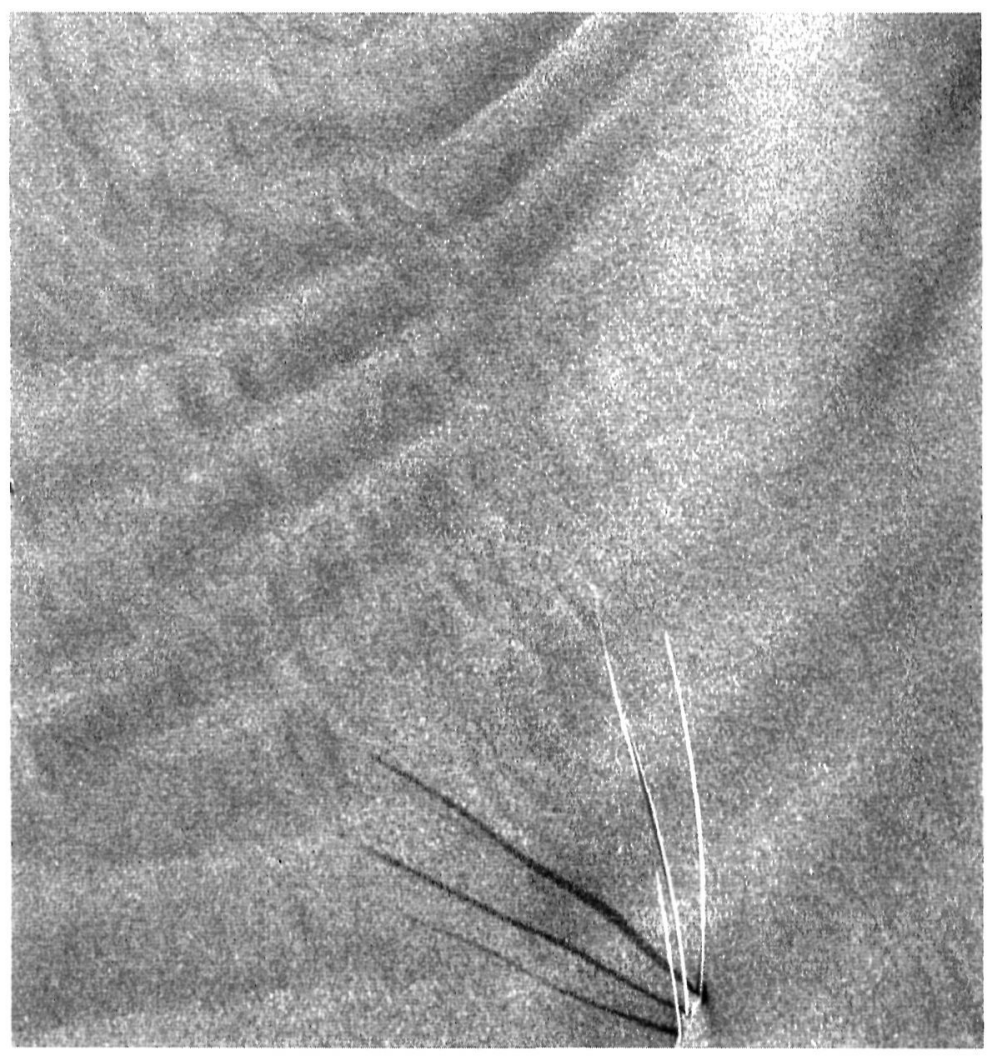

